\title{
Análise comparativa de modelos de alinhamento estratégico
}

\author{
Vanderli Correia Prieto EPUSP e Mackenzie \\ Marly Monteiro de Carvalho EPUSP \\ Adalberto Américo Fischmann FEA USP
}

\section{RESUMO}

Este trabalho se insere no campo de pesquisa em estratégia organizacional e apresenta uma revisão e análise comparativa dos modelos de alinhamento estratégico. A relevância deste estudo está relacionada à importância da execução da estratégia no contexto competitivo e, ao mesmo tempo, à dificuldade enfrentada, devido à complexidade do processo de implementação da estratégia. Foram feitas buscas em periódicos acadêmicos relevantes com a proposta de identificar modelos teóricos que pudessem servir de referência para o processo de alinhamento estratégico. Quatro modelos foram selecionados e analisados utilizando-se o método de análise de conteúdo. A análise comparativa pautou-se em quatro componentes: visão do alinhamento, modelo para implementação, elementos do modelo e processos gerenciais para implementação. Em cada modelo identificam-se diferentes visões sobre como desenvolver o alinhamento, sobre quais elementos e processos gerenciais devem ser considerados. A pesquisa amplia o conhecimento sobre os fatores que contribuem para a eficácia do processo de alinhamento e sobre como desenvolvê-los, além de identificar lacunas na literatura.

\section{Comparative analysis of strategic alignment models}

\begin{abstract}
This work refers to the organizational strategy research field, in which a review and comparative analysis of the strategic alignment models is presented. The relevance of this study is related to the importance of the strategy execution in the competitive context, as well as the difficulties faced due to the complexity of the strategic implementation process. Searches were performed into relevant academic journals in order to identify theoretical models that could be useful as a reference to the strategic alignment process. Four models were selected and analyzed based on the content analysis approach. The comparative analysis was deployed into four components: vision of alignment, model for implementation, elements of the model, and managerial procedures for implementation. In each model was identified different vision on how to develop alignment, and which elements and managerial procedures must be considered. The research enhanced the understanding of the factors that contribute to the alignment process effectiveness and how to develop them, besides it identifies gaps in the literature.
\end{abstract}

\section{KEY WORDS}

Strategic management, organizational strategy (models), organizational effectiveness, strategic planning, organizational performance. 


\section{INTRODUÇÃO}

Estudos recentes apontam que a execução bem-sucedida da estratégia é considerada pelos executivos como uma prática relevante para o bom desempenho organizacional. Em particular, a pesquisa de Nohria et al. (2003) junto a 160 empresas, durante um período de dez anos, revela que, dentre mais de 200 práticas administrativas, a execução da estratégia foi destacada como uma das quatro que são críticas para o sucesso empresarial.

Nesse contexto, o alinhamento é um conceito central retratado pelas pesquisas na área de estratégia, que representam, em linhas gerais, dois eixos sobre a questão da vantagem competitiva. O primeiro eixo aborda a questão da vantagem competitiva com ênfase no poder do mercado, sendo o alinhamento uma condição inerente ao ajuste da estratégia às oportunidades e ameaças do ambiente de atuação da empresa (PORTER, 1979). Já o segundo eixo foca na visão da empresa baseada em recursos (Resourced Based View - RBV), cuja ênfase do alinhamento recai primeiramente sobre os fatores internos (BARNEY, 1991; COLLIS; MONTGOMERY, 1995; WERNERFELT, 1984).

Embora o conceito de alinhamento ou ajuste tenha suas origens nas ciências naturais, no modelo ecológico e na teoria da contingência, ele também aparece como tema central em várias disciplinas (GALBRAITH; KAZANJIAN, 1986; POWELL, 1992; SEMLER, 1997; VENKATRAMAN; CAMILLUS, 1984). Por exemplo, no campo das disciplinas voltadas à gestão das organizações, pode-se encontrar o tema de alinhamento na gestão por objetivos (management by objectives - MBO) e no gerenciamento pelas diretrizes, conhecido pelos nomes que os originaram, hoshin kanri ou policy deployment, com aplicação na área de gestão da qualidade.

No campo da estratégia, o conceito de alinhamento é um requisito presente tanto no processo de formulação quanto de implementação da estratégia (HREBINIAK; JOYCE, 2001; VENKATRAMAN; CAMILLUS, 1984;). Na formulação o conceito refere-se ao ajuste da estratégia ao ambiente competitivo e também está presente na ideia do consenso entre os estrategistas quanto ao resultado das decisões estratégicas, o que irá influenciar o grau de comprometimento e impulsionar a comunicação da estratégia aos demais níveis hierárquicos (EISENHARDT, 1999; FLOYD; WOOLDRIDGE, 1992). Na implementação é requerida a integração entre vários elementos, em especial o alinhamento da estrutura, pessoas, sistema de informações, métricas e recompensas para viabilizar a proposta estratégica.

Se, por um lado, a literatura e a prática organizacional apontam para a importância do alinhamento, autores como
Cypher, (1996), Naman e Slevin (1993), Prieto e Carvalho (2004a, 2004b), Semler (1997), Venkatraman e Camillus (1984) concordam que há lacunas para que o conceito possa ser adequadamente aplicado.

Venkatraman e Camillus (1984) assumem esta lacuna especificamente no campo da estratégia, em que coexistem diferentes perspectivas para a aplicação do conceito de alinhamento dependendo das escolas de pensamento que o empregam. Dada sua complexidade, o conceito de alinhamento é tratado e investigado segundo construtos distintos, de alinhamento externo (formulação da estratégia) interno (implementação da estratégia) e integrado. Existem ainda as classificações quanto ao conteúdo, elementos a serem alinhados com a estratégia, ou quanto ao processo, como realizar o alinhamento.

Há um consenso na literatura de que a prioridade de pesquisadores e executivos tem sido a formulação em detrimento da implementação da estratégia (ATKINSON, 2006; BOSSIDY; CHARAN, 2005; HREBINIAK; JOYCE, 2001). Em consequência, os executivos se ressentem da dificuldade de gerenciar e controlar as diversas atividades de implementação, e os interesses e conflitos de tantos envolvidos, em diferentes funções ou organizações.

Destaque-se que a essência da estratégia é manter as organizações competitivas em meio às descontinuidades, o que implica um processo de implementação dinâmico e que envolve a coordenação de múltiplas variáveis e períodos de tempo mais extensos do que os despendidos no processo de formulação. Neste sentido, atribui-se aos modelos de alinhamento interno a finalidade de assegurar que as atividades necessárias à implementação da estratégia sejam ordenadas de maneira lógica, e integradas e levem ao sucesso na atividade de execução (HREBINIAK; JOYCE, 2001; PRIETO, 2006).

Este trabalho visa identificar modelos teóricos que possam servir como referência para o processo de alinhamento interno, bem como as ações ou etapas que fazem parte desse processo. A delimitação do trabalho se deu considerando-se que as diferentes classificações de alinhamento, interno e externo, apresentam uma grande diversidade das variáveis a serem investigadas e, ainda, diante da lacuna identificada na literatura quanto à dificuldade de operacionalização do conceito de alinhamento estratégico, em particular o alinhamento interno, no qual as lacunas são mais significativas.

$\mathrm{O}$ artigo está estruturado em cinco seções. A seção 2 apresenta a síntese da discussão teórica sobre alinhamento estratégico e operacionaliza os construtos da pesquisa. A abordagem metodológica é apresentada na seção 3 , justificando a opção pelo método de análise de conteúdo. $\mathrm{Na}$ seção 4 são apresentados quatro modelos de alinhamento 
estratégico, sua análise distintiva e identificação das lacunas na literatura. Finalmente, a seção 5 traz as conclusões, limitações e recomendações para trabalhos futuros.

\section{FUNDAMENTOS TEÓRICOS E PRINCIPAIS CONCEITOS}

O conceito de alinhamento tem as suas origens nos estudos da abordagem contingencial. As principais influências estão nos estudos sobre o alinhamento entre a organização e o ambiente (BURNS; STALKER, 1961; KATZ; KAHN 1966; THOMPSON, 1967). De acordo com Ginsberg e Venkatraman (1985), o pensamento contingencial destaca que não existe uma única melhor maneira de administrar e que tudo depende das características ambientais relevantes para a organização; a ideia central é o alinhamento dos recursos organizacionais às oportunidades e ameaças do ambiente (ANDREWS, 1971; CHANDLER, 1962). Outro alinhamento relevante é entre a tecnologia e a estrutura (WOODWARD, 1965). Adicionalmente, os contingencialistas estabeleceram, também, a conexão entre o alinhamento e a performance organizacional, que, em geral, apresenta uma correlação positiva, ou seja, a boa performance das organizações está associada a um alinhamento adequado entre as variáveis endógenas (estratégia, estrutura e processos gerenciais) e as variáveis exógenas (incerteza ambiental e tecnologia).

Outra corrente influente na literatura sobre alinhamento é o pensamento sistêmico, aliado ao conceito de sistemas abertos originário dos estudos de Bertalanffy, nos anos 1950. Nessa abordagem, a organização é considerada como um organismo vivo, que influencia e é influenciada pelo ambiente, o que exige capacidade de adaptação e equilíbrio. Adaptação no sentido de que mudanças internas serão necessárias para atender às demandas do ambiente externo, enquanto que o equilíbrio visa manter o grau de coesão entre as dimensões internas.

$\mathrm{Na}$ literatura sobre estratégia o conceito de alinhamento pode assumir o sentido de agrupamento, coesão, ajuste, congruência entre diferentes dimensões (GALBRAITH; KAZANJIAN, 1986).

O termo mais utilizado para abordar o alinhamento estratégico externo é ajuste (fit). O conceito de alinhamento externo está presente na literatura referindo-se ao ajuste das capacidades, recursos e estratégia ao ambiente competitivo da organização. Mais precisamente, "fit" tem sido definido como o grau de ajuste da organização ao ambiente (NAMAN; SLEVIN, 1993).
As pesquisas sobre alinhamento externo têm sido conduzidas em termos de integração de capacidades (FUCHS et al., (2000); alinhamento dinâmico e inércia organizacional (SIGGELKOW, 2001; ZAJAC, et al. 2000), formulação da estratégia e desempenho organizacional (DESS, 1987), dentre outras.

Já o alinhamento estratégico interno pode assumir diferentes denominações na literatura, como implementação da estratégia, coesão e coerência corporativa. Essa classificação refere-se à mobilização dos recursos internos, tangíveis ou intangíveis, para implementação da estratégia formulada. As suas raízes teóricas estão na literatura de implementação da estratégia e de mudança organizacional (STEPANOVICH; MUELLER, 2002; VENKATRAMAN; CAMILLUS, 1984). A proposição de Chandler (1962) sobre o alinhamento da estrutura à estratégia é seminal neste campo de estudos. Também são relevantes os trabalhos que surgiram nos anos 1970 com a visão da organização baseada em recursos (RBV), com destaque para Galbraith (1977), Peters e Waterman (1982) e Miles e Snow (1978). Neste contexto, os elementos chave a serem alinhados incluem a estratégia, as pessoas, a estrutura e a gestão dos processos.

\section{processo de alinhamento estratégico é definido como o exercício prático de um conjunto de atividades estruturadas com a finalidade de realizar o alinhamento estratégico.}

Para Miles e Snow (1984), o alinhamento estratégico é visto tanto como um processo quanto como um resultado, em busca do ajustar dinâmico da organização ao seu ambiente externo e aos recursos internos. Segundo os autores, "organizações de sucesso realizam o alinhamento estratégico em relação ao seu mercado de atuação e suportam as suas estratégias com estruturas e processos gerenciais adequados".

Os estudos geralmente abordam a necessidade de manter os recursos da organização internamente alinhados durante a implementação da estratégia, em particular, em tempos de mudança estratégica, conforme destacado nos trabalhos de Kilman (1989) desenvolvidos em ambientes de fusão e aquisição

O consenso estratégico é também uma das dimensões do alinhamento interno que sobressaem na literatura e refere-se à concordância, ao consenso de opiniões que se estabelece tanto na formulação, quanto na implementação da estratégia, representado pelos trabalhos de Dess (1987), Floyd e Wooldridge (1992), Holmburg et al. (1999) e Priem (1990). 
O desempenho da organização é altamente dependente tanto do alinhamento interno como do externo e o processo de alcançar ambos os alinhamentos é dinâmico e precisa ser continuamente monitorado e ajustado (LABOVITZ; ROSANSKY, 1967; SIGGELKOW, 2001).

Contudo, uma característica dos estudos de alinhamento é que as bases teóricas traçadas e os principais construtos e variáveis para o alinhamento interno e externo são distintos, conforme destacado por Prieto (2006), Prieto e Carvalho (2004a, 2004b), Stepannovich e Mueller (2002) e Venkatraman e Camillus (1984). O alinhamento externo considera a perspectiva da formulação da estratégia e, primariamente, $o$ ajuste da estratégia às variáveis ambientais. Em contraste, o foco do alinhamento interno é a implementação e o ajuste entre a estratégia e as variáveis internas. pergunta para o que a organização precisa fazer para ter sucesso com uma estratégia já determinada. Estando a estratégia definida, o problema reside na necessidade de escolher uma forma para alcançar os objetivos propostos.

Neste contexto, a literatura propõe ferramentas para medir o grau de alinhamento estratégico, que conduzam a organização a um estágio de controle da estratégia (KAPLAN; NORTON, 1996; FUCHS et al., 2000; LABOVITZ; ROSANSKY, 1997).

Por outro lado, outros autores, como Campbell e Alexander (1997) e Hambrick e Cannella (1989) apresentam argumentos contrários à distinção entre formulação e implementação. Para estes autores, apesar de a média gerência não ter necessariamente o perfil requerido de um estrategista, o conhecimento das operações diárias e a gestão de linha de frente do negócio são fontes importantes para a inovação estratégica, sendo que a probabilidade destas idéias emergirem é maior no processo criativo de formulação da estratégia. Outros argumentos dizem respeito à necessidade de planejar e analisar os ajustes necessários à implementação já na fase de formulação para ter certeza de que a estratégia é viável.

Em ambientes turbulentos, a estratégia

\subsection{Implementação de estratégias organizacionais}

Conforme já destacado, o alinhamento é ao mesmo tempo um resultado e um processo (MILES; SNOW, 1984). Um processo é comumente definido como um conjunto de tarefas e atividades que contribui para transformar entradas em saídas. No caso do alinhamento enquanto processo, o conceito refere-se ao exercício prático de um conjunto de atividades estruturadas com a finalidade de realizar o alinhamento estratégico (PRIETO, 2006). O exercício do alinhamento interno deve levar à implementação bem-sucedida da estratégia.

A formulação e a implementação são claramente relacionadas e interdependentes, e fazem parte de um mesmo processo: o processo estratégico. Entretanto, cada uma destas atividades pode ser diferenciada e discutida separadamente, conceitualmente e na prática.

$\mathrm{Na}$ lógica, a implementação segue a formulação. A ideia de que para promover a implementação é necessário ter uma estratégia claramente definida está presente na literatura acrescida de argumentos sobre os fatores e os processos que favorecem ou não a sua implementação. Bower (1982), por exemplo, ressalta que enquanto o processo de formulação da estratégia foca no que a empresa pode fazer de melhor para enfrentar o ambiente competitivo no qual está inserida, o processo de implementação da estratégia muda o foco da pode ser modificada devido ao próprio processo de aprendizado e crescimento que acontece quando a estratégia é colocada em ação. Nesse contexto a estratégia enquanto processo assume maior relevância.

Para Mintzberg (1994) é necessário construir um entendimento de que a estratégia não é necessariamente um exercício formal, pois sua essência é a concretização da estratégia por meio das ações. Em outras palavras, a estratégia é elaborada para ser concretizada, mas a prática da implementação também pode levar a mudanças estratégicas. Deve-se, portanto, considerar não só o intencional, mas também as estratégias emergentes, o que atribui ao processo estratégico a característica de inovação e aprendizado.

Eisenhardt (1999) destaca ainda o caráter intuitivo do processo estratégico e advoga uma abordagem por regras simples que permita o rápido ajuste em ambientes turbulentos (EISENHARDT; SULL, 2001). Deriva desse cenário a necessidade de constante revisão, o que dá a característica dinâmica ao processo estratégico (CHRISTENSEN, 1997; COURTNEY; KIRKLAND; VIGUERIE, 1997)

Na dinâmica deste processo de transformação da estratégia em ação, o melhor plano estratégico não é considerado linear, mas circular, no sentido de que é continuamente avaliada e revisada a maneira como está sendo conduzido, por meio das ações de planejamento, mensuração e ajuste (Mc- 
CONKEY, 1988). Bower (1982) apresenta um entendimento semelhante, quando afirma que o processo de implementação não necessariamente precisa começar com a formulação da estratégia, mas que todas as partes do processo estratégico estão ativas a todo momento.

Diante da complexidade que se impõe, um modelo de alinhamento estratégico tem a finalidade de assegurar que as atividades necessárias ao processo de transformação da estratégia em ação sejam ordenadas de maneira lógica e integrada, sem perder a flexibilidade, permitindo que mudanças estratégicas possam ser incorporadas a este processo.

Corroborando com este raciocínio, Hrebiniak e Joyce (2001) afirmam que para minimizar a complexidade deste processo, a utilização de um modelo de implementação estratégica possibilitaria aos administradores um delineamento lógico das principais categorias de atividades de implementação e das relações entre elas. Teria como vantagem reduzir a uma dimensão tratável e administrável um problema antes extremamente complexo e, mais importante, deduzir consequências e resultados específicos das atividades de implementação (FISCHMANN, 1987).

O modelo integra variáveis que têm sido tratadas separadamente na literatura, mas que precisam ser visualizadas e discutidas em conjunto a fim de aumentar a compreensão do processo total de implementação (HREBINIAK; JOYCE, 2001).

Desta forma, optou-se pela análise de modelos cujo foco seja o alinhamento interno, que se desenvolve no âmbito da implementação da estratégia. Esse recorte metodológico permite reduzir a diversidade das variáveis a serem investi- gadas e auxilia na análise comparativa dos modelos extraídos da revisão de literatura. Além disso, a lacuna de literatura sobre alinhamento interno é maior, conforme já destacado na seção introdutória.

\section{ASPECTOS METODOLÓGICOS}

Conforme exposto na seção introdutória, dada a especificidade e abrangência das variáveis a serem investigadas, as pesquisas sobre alinhamento são conduzidas em termos de alinhamento externo e interno. Seguem a taxonomia dos estudos da área de estratégia, elaborados, respectivamente, em termos de formulação e implementação.

O presente estudo tem como objetivo identificar modelos na literatura que possam servir como referência para o processo de alinhamento estratégico interno. Pretende-se investigar quais ações ou etapas fazem parte do processo de alinhamento. Para tanto, um quadro analítico distintivo destes modelos será elaborado, visando identificar lacunas entre os mesmos. A figura 1 sintetiza a abordagem metodológica utilizada.

Considerou-se um modelo como sendo uma estrutura teórica que pode indicar quais fatores são mais críticos ou importantes em um dado contexto e como tais fatores estão relacionados entre si, conforme definido por Nadler e Tushman (1980). Uma premissa da construção de um modelo é a efetiva organização de suas subpartes ou componentes, que devem ser consistentemente estruturadas e gerenciadas buscando a congruência.

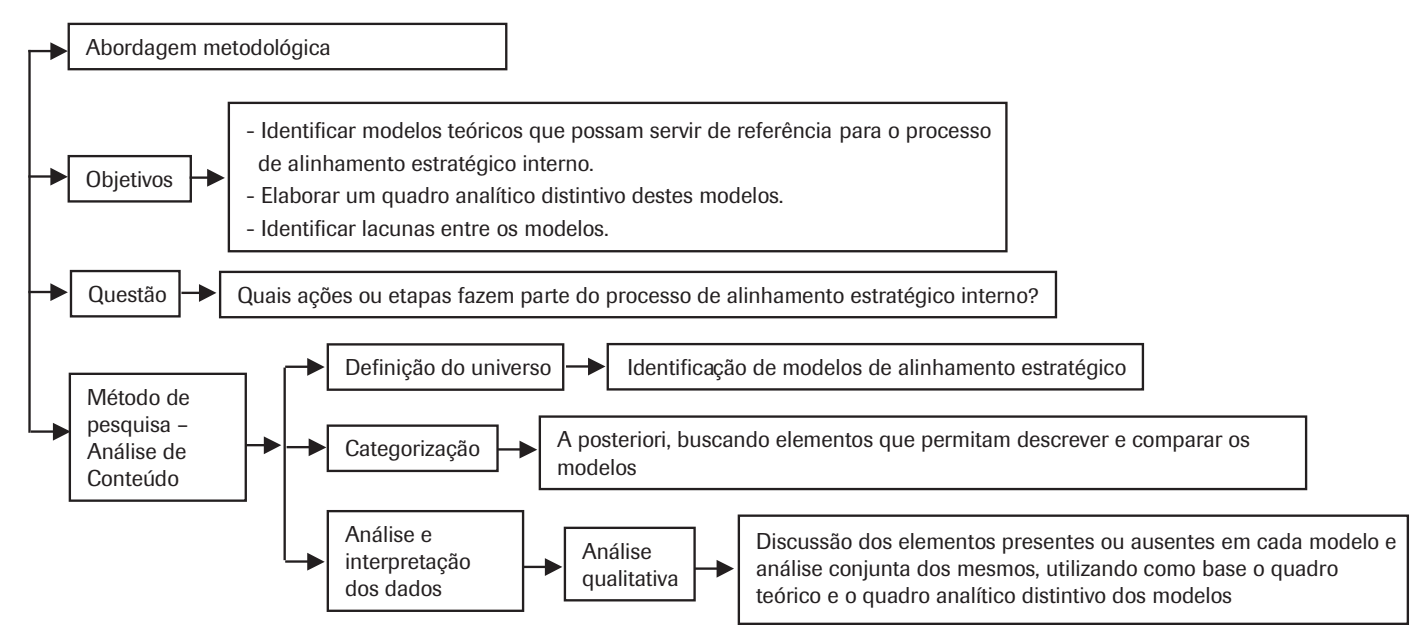

Figura 1: Abordagem metodológica. 
Considerando-se a delimitação do trabalho, a prioridade é a seleção de modelos que tenham uma proposta para alinhamento dos aspectos internos da organização. Este e outros termos relevantes para o entendimento da estrutura deste artigo estão definidos no Quadro 1.

A formulação do quadro-analítico baseou-se no método de pesquisa denominado análise de conteúdo. Segundo Bardin (2000), é necessário definir os limites da análise de conteúdo, o seu campo, onde começa e acaba e com qual finalidade será empregada. Propõe-se que a análise seja organizada cronologicamente em: pré-análise; exploração do material e tratamento; e interpretação dos resultados. As atividades-chave dentro deste processo são: (1) a definição do universo, ou seja, a escolha de documentos, (2) a categorização e (3) a análise e interpretação dos resultados.

Definição do universo: é composta pelos quatro modelos de alinhamento estratégico que serão apresentados e discutidos na próxima seção. Os modelos foram selecionados pela sua adequação à delimitação e ao conceito de alinhamento estratégico interno propostos neste trabalho. A base teórica utilizada para a seleção dos modelos foi construída por meio do levantamento cruzado de referências, o qual foi realizado a partir dos trabalhos de Stepanovich e Mueller (2002) e Prieto e Carvalho (2004a). Foram analisados 40 trabalhos provenientes de periódicos das áreas de administração e negócios e foram encontrados seis modelos de alinhamento estratégico, sendo que dois deles não se adequavam à proposta deste trabalho.

Categorização: esta etapa destina-se a determinar as dimensões que serão analisadas e serve para classificar o conteúdo e deve, normalmente, permitir o aparecimento de variáveis e fatores de influência que se ignoravam no início dos trabalhos. As categorias, quando não se tem uma ideia precisa, devem surgir com base no próprio conteúdo (WEBER, 1990).

$\mathrm{Na}$ análise inicial dos modelos identificou-se que os autores apresentavam diferentes perspectivas sobre como desenvolver o processo de alinhamento estratégico. Assumiu-se que a visão do alinhamento seria um dos elementos para categorização do conteúdo. Em seguida, para cada um dos modelos, foram extraídas dos textos dos autores todas as frases que representavam características dos modelos. O agrupamento e a análise de tais características levaram à compreensão dos componentes que descrevem os modelos: (1) visão do alinhamento, (2) modelo para implementação da estratégia, (3) elementos do modelo e (4) processos gerenciais desenvolvidos para promover o alinhamento estratégico.

Análise e interpretação dos resultados: será elaborado um quadro analítico visando a comparação e identificação de lacunas entre os modelos. A análise será qualitativa, por meio da discussão dos elementos presentes ou ausentes em cada modelo, utilizando como base o quadro teórico.

\section{APRESENTAÇÃO DOS MODELOS DE ALINHAMENTO ESTRATÉGICO}

Foram identificados quatro modelos de alinhamento estratégico interno, por meio do processo de busca e revisão da literatura:

- Modelo 1 - Labovitz e Rosansky (1997)

- Modelo 2 - Balanced scorecard: Kaplan e Norton (1992, 1993, 1996, 2000)

- Modelo 3 - Hambrick e Cannella (1989)

- Modelo 4 - Organizational Fitness Profiling (OFP): Beer e Eisenstat $(1996,2000)$

\section{Quadro 1: Definição dos termos-chave do artigo.}

Alinhamento estratégico: tanto um processo como um resultado - uma dinâmica que procura ajustar a organização externamente em relação ao seu ambiente de atuação, por meio da formulação da estratégia (alinhamento externo) e, internamente, buscando um todo coeso entre a estratégia, os recursos e os processos gerenciais (alinhamento interno).

Aspectos internos da organização: refere-se aos elementos a serem alinhados internamente, definidos como estratégia, recursos e processos gerenciais, e que englobam, por exemplo, a estratégia, a estrutura, processos, pessoas, clientes, habilidades, liderança, sistemas de remuneração, sistemas de informação e os processos gerenciais. Cada uma das dimensões precisa ser consistente com a estratégia e umas com as outras.

Modelo de alinhamento estratégico: representação sistemática de um conjunto de princípios e ações com a finalidade de promover o alinhamento estratégico.

Processo de alinhamento estratégico: exercício prático de um conjunto de atividades estruturadas com a finalidade de realizar o alinhamento estratégico.

Processo de alinhamento estratégico interno: exercício prático de um conjunto de atividades estruturadas com a finalidade de promover a implementação da estratégia. 
Nesta seção será feita uma breve apresentação destes modelos.

\subsection{Modelo 1 - Labovitz e Rosansky}

Labovitz e Rosansky (1997) vêm o alinhamento como um processo de integração dos elementos da organização em torno do propósito essencial dos negócios, denominado por eles "the main thing". Não se apresenta uma definição precisa para o termo, a proposta é a elaboração de uma estratégia ampla, um diferencial dos negócios capaz de fazer com que as pessoas tenham uma direção única com um propósito compartilhado. Os elementos a serem alinhados são a estratégia e pessoas (alinhamento vertical) e clientes e processos (alinhamento horizontal) - ver Figura 2.

O alinhamento no sentido vertical trata da relação entre a definição da estratégia de negócios da empresa e a efetiva concretização da estratégia pelas pessoas.

O modelo considera o sistema de métricas a chave para a realização do alinhamento vertical, aliado a recompensas e reconhecimento. $\mathrm{O}$ desdobramento da estratégia requer a implementação de indicadores críticos de sucesso, metas e atividades, definidos a partir do propósito essencial, e que devem ser levados a todos os níveis hierárquicos da organização.

O alinhamento no sentido horizontal é um alinhamento entre clientes e processos. A dimensão horizontal envolve a compreensão das necessidades do cliente e o alinhamento dos processos (interfuncionais) capazes de entregar o que o cliente necessita. A ideia também é identificar quais são os clientes alvo deste processo - os clientes certos.

Labovitz e Rosansky (1997) propõem uma ferramenta específica para diagnosticar o grau de alinhamento, que per-

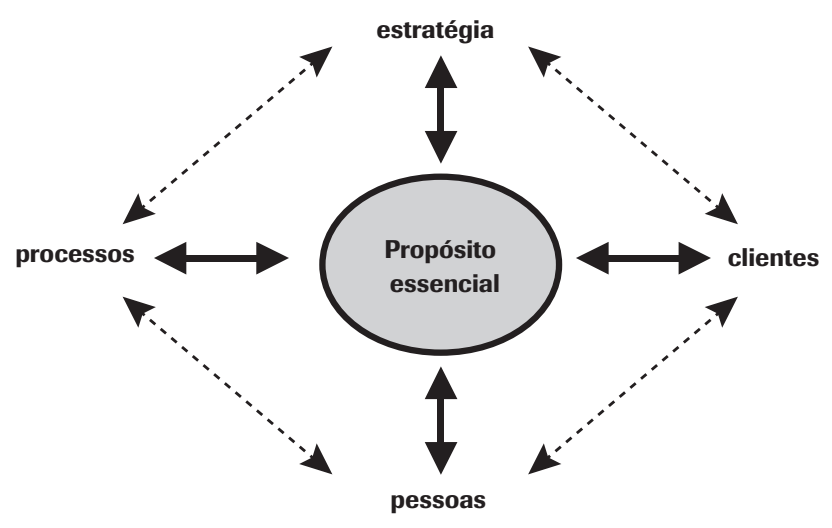

Figura 2: Modelo 1 - Labovitz e Rosansky.

Fonte: Adaptado de Labovitz e Rosansky, 1997, p. 44. mite traçar o perfil de alinhamento da organização. A ideia é de um autodiagnóstico; a própria organização pode aplicar, internamente, um questionário proposto pelos autores. O resultado será uma medida visual e quantitativa do grau de alinhamento entre a estratégia, as pessoas, os processos e os clientes.

\subsection{Modelo 2 - Balanced scorecard (BSC)}

A base para a elaboração do BSC é a definição ou revisão da estratégia da empresa, que deve considerar os fatores críticos de sucesso para atuação no segmento de negócios no qual a empresa está inserida. O modelo considera, então, a estratégia já formulada, a qual será desdobrada em um scorecard multidimensional, integrando, além das medidas de desempenho financeiro, medidas de desempenho relacionadas às perspectivas dos clientes, dos processos internos $\mathrm{e}$ de aprendizado e crescimento (KAPLAN; NORTON, 1992, 1993, 1996 e 2000). A Figura 3 apresenta o modelo.

Enquanto sistema de alinhamento estratégico, o modelo apoia-se em dois eixos principais, o da comunicação e do controle da estratégia. A proposta do BSC é disseminar o conhecimento a respeito da estratégia por todos os níveis e áreas da organização por meio do mapa estratégico. A dinâmica de controle da estratégia acontece pela definição de medidas de desempenho equilibradas, indicadores de tendência (leading) e de resultado (lagging), financeiras e não financeiras, que permitem acompanhar o desempenho dos negócios no curto e no longo prazo.

\subsection{Modelo 3 - Hambrick e Cannella}

Na visão de Hambrick e Cannella (1989), o alinhamento é visto como um processo gerencial a ser conduzido pelo estrategista. Apoia-se em um processo de negociação da estratégia, interna e externamente à organização, buscando eliminar obstáculos, promover ajustes e convencer a respeito da importância da estratégia. O modelo para realizar o alinhamento caracteriza-se pela definição das etapas a serem conduzidas pelo executivo, desde a formulação até a implementação da estratégia. Uma das ideias centrais destes autores é que a implementação da estratégia começa na formulação. Consideram que um sinal de uma estratégia bem formulada é ser implementável e que separar as fases de formulação e implementação é a raiz para muitas falhas na execução.

O modelo envolve três etapas (ver Figura 4). A primeira, é considerada uma "preparação de terreno" para a nova estratégia e inclui ter uma amplitude de entradas para a formulação da estratégia e cuidar dos obstáculos de implementação. A segunda etapa consiste em tratar das influências que são relevantes para a implementação, 
segundo cinco aspectos: comprometimento dos recursos, políticas e programas das subunidades, estrutura, recompensas e pessoas. A terceira etapa é o convencimento a respeito da estratégia, envolvendo todos os níveis, dentro e fora da organização. Trata-se do papel do estrategista de construir e manter o suporte necessário para a implementação da estratégia em meio às resistências que naturalmente surgem devido ao interesse de determinadas partes em manter a velha estratégia ou por falta de compreensão da nova proposta.

\subsection{Modelo 4 - Organizational Fitness Profiling (OFP)}

Beer e Eisenstat $(1996,2000)$ desenvolveram uma ferramenta que foi primeiramente denominada Strategic Human Resource Management (SHRM) e depois Organizational Fitness Profiling (OFP), respectivamente, em duas publicações: 1996 e 2000.

O alinhamento é considerado um processo de mudança que envolve o aprendizado da própria organização a respeito da reformulação da estratégia e do realinhamento constante.

O modelo proposto (Figura 5) é o diagnóstico de ajuste organizacional que visa à identificação de barreiras ao alinhamento, o qual deve ser seguido da elaboração de um plano para eliminar as deficiências encontradas. Quatro questões-chave orientam o diagnóstico:

Na caixa 1 questionam-se quais são as deficiências em capacidades que podem afetar a performance financeira, a

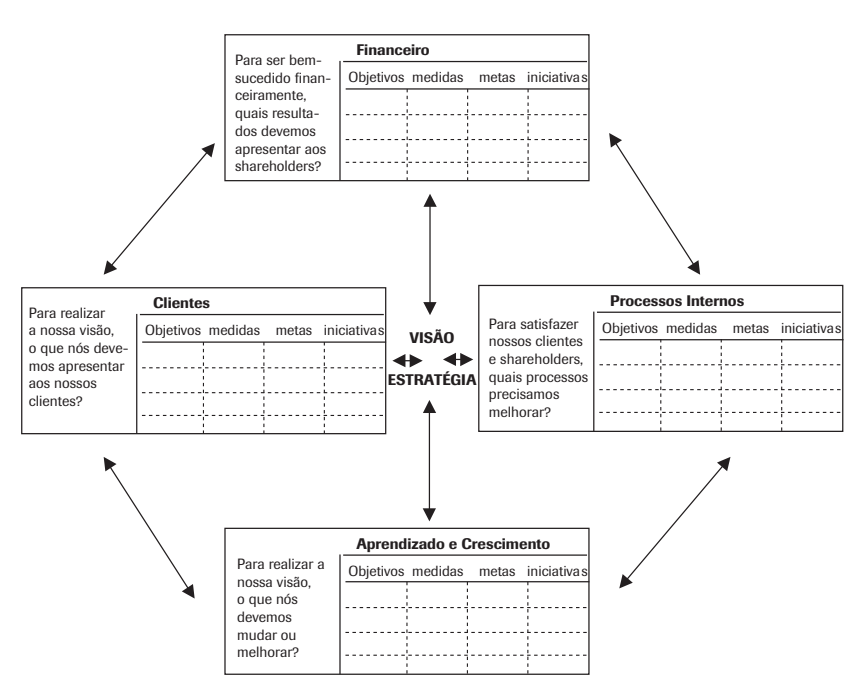

Figura 3: Modelo 2 - Balanced scorecard.

Fonte: Adaptada de Kaplan e Norton, 1996. satisfação dos clientes e a satisfação dos empregados, representados na caixa 2 .

As capacidades organizacionais demandam que a liderança coloque em prática as habilidades de coordenação entre as partes da organização, obtenha o comprometimento das pessoas e dos grupos para a realização das tarefas estratégicas e desenvolva competência técnica e interpessoal para solucionar problemas e coordenar soluções. A satisfação dos stakeholders é condição para a sobrevivência da organização.

$\mathrm{Na}$ caixa 3 busca-se diagnosticar como as forças e as deficiências nas capacidades organizacionais poderiam afetar o sucesso na implementação das alternativas estratégicas.

Além disso, identificam-se quais forças contextuais (caixa 4) - como políticas corporativas e comportamento da alta administração (no caso de divisões de grandes empresas) bem como as forças competitivas ou do mercado de trabalho podem causar os problemas.

Conclui-se o diagnóstico investigando os aspectos da estrutura organizacional (caixa 5) que podem estar causando as deficiências identificadas na capacidade organizacional.

Uma das condições para a aplicação do modelo é um ambiente participativo, uma vez que todo o processo é conduzido por uma força-tarefa composta para este fim, que conduz o processo internamente e apresenta e discute os resultados com a alta administração.

\section{DISCUSSÃO DOS RESULTADOS}

Nesta seção faz-se a análise comparativa dos modelos a partir de quatro componentes, seguida de uma análise geral dos modelos face aos principais construtos de alinhamento estratégico.

\subsection{Análise comparativa dos modelos de alinhamento}

Os modelos foram submetidos ao método da análise de conteúdo com o objetivo de permitir a análise comparativa e a identificação de lacunas. Aplicou-se a categorização proposta na seção de metodologia para a descrição dos modelos: (1) visão do alinhamento, (2) modelo para implementação da estratégia, (3) elementos do modelo e (4) processos gerenciais desenvolvidos para promover o alinhamento estratégico. Os resultados estão demonstrados no Quadro 2.

Foram identificadas quatro diferentes visões a respeito do alinhamento: (1) como um processo de integração dos recursos e sistemas da organização em torno do propósito essencial dos negócios; (2) como processo de desdobramento da estratégia em indicadores de desempenho; (3) como processo de negociação e convencimento a respeito da estratégia; (4) como processo de mudança. 
Nota-se no Quadro 2 que essas visões sobre o alinhamento apresentam duas vertentes, a da seleção e a da exclusão dos elementos e processos gerenciais que devem compor o modelo de alinhamento. Uma análise comparativa dos modelos permite identificar, então, as principais lacunas.

Os modelos 1 e 2 apoiam-se em um sistema de métricas para alinhamento estratégico. Ambos especificam clientes, processos e pessoas como dimensões a serem alinhadas. $\mathrm{O}$ aprendizado e crescimento (modelo 2) não são enfatizados no modelo 1, assim como finanças. Neste sentido, as perspectivas do modelo 2 são mais abrangentes, uma vez que a perspectiva de aprendizado e crescimento trabalha com as condições de sobrevivência da empresa no médio e longo prazo. Esses modelos têm a estratégia como base para o desdobramento dos indicadores de desempenho.

O modelo 2 não enfatiza parâmetros para a definição da estratégia que sirvam de base para o processo de alinhamento interno. Esta definição acontece no modelo 1, ainda que não seja aprofundada, quando trata do propósito essencial.

No modelo 2 o alinhamento ocorre por meio da interdependência entre os indicadores quando da construção do mapa estratégico. Já no modelo 1 , o alinhamento ocorre por meio do desdobramento hierárquico dos indicadores de desempenho. $\mathrm{O}$ modelo 2 mantém a coesão entre as quatro perspectivas, o que não fica claro no modelo 1 .

Não é uma prioridade do modelo 2 abordar os aspectos intangíveis presentes em um processo de alinhamento. É uma prioridade do modelo incluir tais aspectos no sistema de métricas. O modelo 1 admite a influência de tais aspectos, principalmente liderança e cultura, mas também não trata de como desenvolvê-los.
Os modelos 3 e 4 são mais explícitos ao abordar os aspectos intangíveis presentes em um processo de alinhamento estratégico. No modelo 4, o levantamento de barreiras à implementação da estratégia acontece junto às pessoas no ambiente interno à organização, ao contrário do que propõe o modelo 3. Uma lacuna do modelo, portanto, é não considerar que os obstáculos à implementação da estratégia podem estar no ambiente externo e podem não ter sido percebidos por pessoas internas à empresa.

O foco do modelo 4 é diagnosticar as barreiras que possam comprometer a implementação de uma estratégia que se encontra em ação. Neste sentido, comparando-se com o modelo 3, identifica-se uma lacuna neste modelo, de ser reativo e não proativo no levantamento de barreiras à implementação da estratégia. No modelo anterior tem-se a visão de evitar as barreiras à estratégia por meio do processo de negociação e convencimento.

Não é uma prioridade dos modelos 3 e 4 definir uma estrutura para desdobramento da estratégia, como é apresentado nos modelos 1 e 2, por meio da construção dos mapas estratégicos.

Identificam-se nos modelos diferentes processos gerenciais para implementação da estratégia e, inerentemente, diferentes habilidades para colocá-los em ação. Nos modelos 1 e 2, o sistema de métricas é a chave para o desenvolvimento dos processos gerenciais de planejamento, implementação e revisão da estratégia. Requer, principalmente, a habilidade de construir, comunicar e gerenciar os indicadores de desempenho. Entende-se que os mesmos processos estão compreendidos no modelo 3 , porém a chave para o seu desenvolvimento é a capacidade de articulação e planejamento

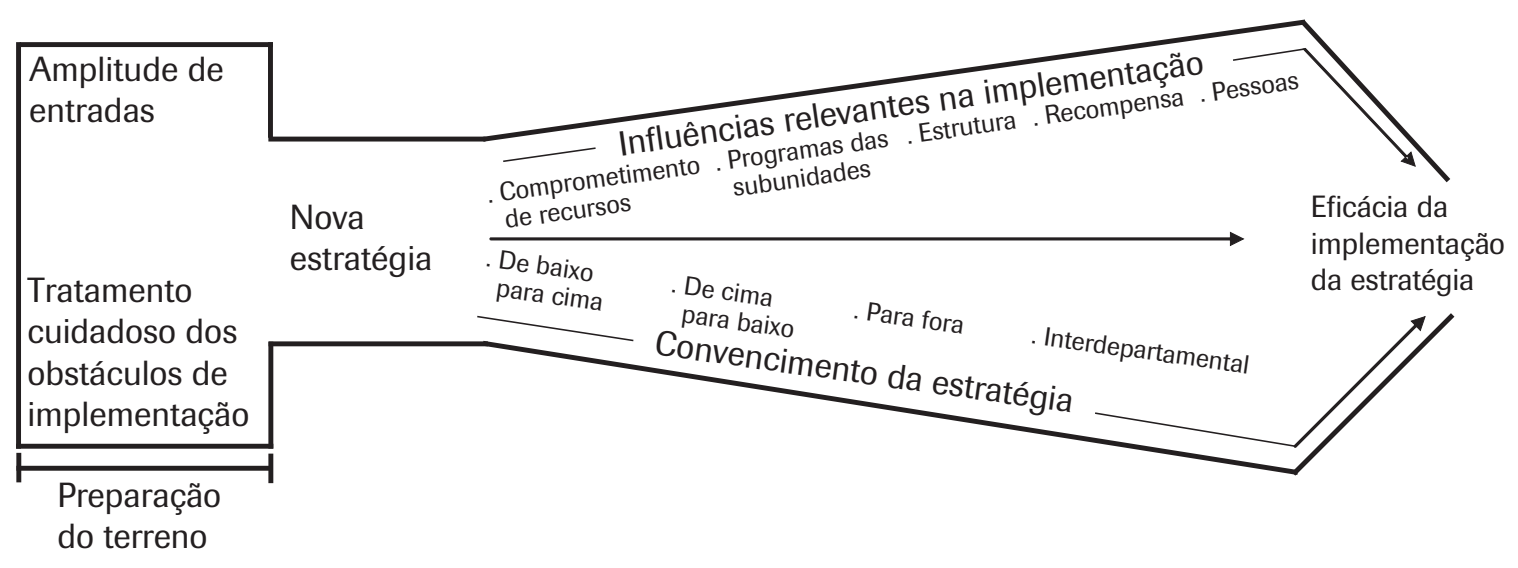

Figura 4: Modelo 3 - Hambrick e Cannella.

Fonte: Adaptada de Hambrick e Cannella, 1989. 
do estrategista. No modelo 4, a base para o desenvolvimento dos processos gerenciais é o diagnóstico realizado em um clima de diálogo amplo, que requer a habilidade, ou coragem (BEER; EISENSTAT, 2004) de publicamente confrontar as barreiras à implementação da estratégia.

Ora os modelos tendem a priorizar aspectos tangíveis (modelos 1 e 2), ora intangíveis (modelos 3 e 4) presentes em um processo de alinhamento estratégico.

No modelo 3, o processo de alinhamento estratégico assume uma característica proativa - não evidenciada com a mesma ênfase nos outros modelos - devido ao planejamento da implementação da estratégia durante a fase de formulação e pela forma com que propõe a identificação e tratamento de barreiras à implementação em todo o processo.

Os modelos também se diferenciam pela proposta da identificação de barreiras à implementação da estratégia (modelos 3 e 4), diagnóstico de alinhamento (modelos 1 e 4), determinação do papel do estrategista na implementação (modelo 3 ) e por promover um questionamento das capacidades, inclusive dos executivos, nas escolhas estratégicas (modelo 4).

\subsection{Fatores-chave para o alinhamento estratégico}

Os modelos selecionados estão de acordo com a definição de alinhamento proposta neste trabalho (ver Quadro 1). Identifica-se um processo de alinhamento em termos de

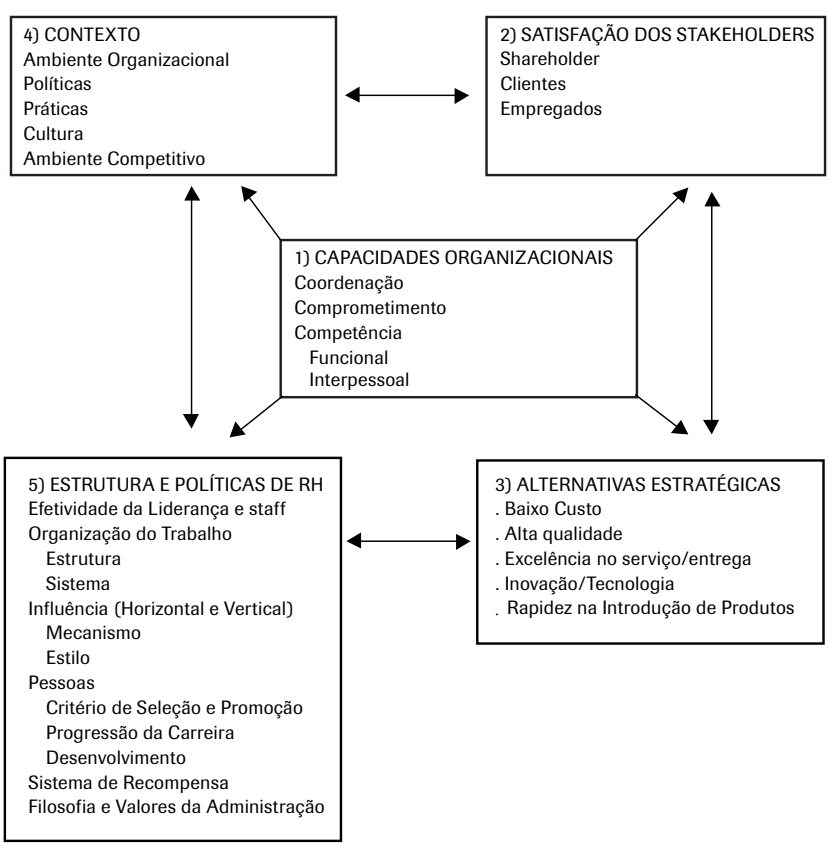

Figura 5: Modelo 4 - Beer e Eisenstat.

Fonte: Adaptada de Beer e Eisenstat, 1996. elementos a serem ajustados e processos gerenciais a serem conduzidos, com o objetivo de obter o resultado previsto na implementação da estratégia.

A identificação de lacunas entre os modelos deixa explícitas as limitações individuais de cada um deles, ao mesmo tempo em que amplia a compreensão sobre o conceito de alinhamento estratégico interno.

Uma análise conjunta dos modelos identificados (Quadro 2) com os principais construtos de alinhamento identificados na literatura (ver seção 2) permite identificar lacunas teóricas a serem desenvolvidas, bem como buscar a complementaridade entre os modelos, aumentando a cobertura do tema. Para promover essa análise foram considerados quatro imperativos consolidados pela literatura (ver Quadro 3): ambiente, estrutura/sistema, liderança e estratégia (MILLER, 1987).

Pode-se observar no Quadro 3 que os modelos identificados focam em aspectos relacionados a estrutura/sistemas e liderança. Por um lado, essa característica é derivada do foco em modelos de alinhamento estratégico interno, em que o ajuste externo (formulação), em parte, é assumido como realizado.

No entanto, a literatura sobre implementação de estratégia é enfática em salientar a característica dinâmica do processo de alinhamento, que envolve inovação e aprendizado, e pode levar a mudanças estratégicas (MINTZBERG, 1994, EISENHARDT, 1999, EISENHARDT; SULL, 2001). Desta forma, os modelos de alinhamento interno identificados, embora adotem a lógica de que a implementação segue a formulação, devem também conceber que os aspectos endógenos também têm força para influenciar e modificar as estratégias e o ambiente, e que o modelo de implementação deve manter canais abertos de realimentação. Apenas o modelo 3 assume uma característica mais proativa, com canais atrelados à formulação da estratégia e sua revisão com base no tratamento de barreiras à implementação em todo o processo.

De maneira geral, os modelos enfatizam, com relação à estratégia, o foco nos interesses dos stakeholders, aspecto, em geral, monitorado por indicadores e relacionado às premiações. Outro aspecto enfatizado nos modelos selecionados é o quão compreensível a estratégia é para aqueles que vão colocá-la em ação. A literatura considera que a definição de objetivos que tenham significado para as pessoas não é uma tarefa tão simples e não pode ser confundida com a simples declaração, sem a devida apropriação pela organização, de termos como visão, missão, intenção estratégica, objetivos dos acionistas e foco no cliente. Campbell e Alexander (1997) sugerem que a chave para o desenvolvimento de uma boa estratégia está na conscientização de seus benefícios pelos executivos. É necessário que os executivos tenham clareza do composto de valor proposto pela organização, sua distinção 
face aos concorrentes, os segmentos de mercado almejados e, em especial, as escolhas feitas e os trade-offs que ela exige (PORTER, 1996) .

Quanto ao imperativo ambiente, os modelos são vagos e retornam à temática do interesse dos stakeholders, mas com a perspectiva de gerenciamento das mudanças na estratégia. Os modelos buscam estabelecer canais de informação que levem à revisão da estratégia formulada e também ao tratamento de possíveis obstáculos a mudanças na estratégia, advindos da fase de implementação. Para tanto, propõem, em geral, diagnósticos das capacidades organizacionais, em uma linha aderente à corrente da visão da empresa baseada em recursos (BARNEY, 1991; COLLIS; MONTGOMERY, 1995; WERNERFELT, 1984).
Os modelos dedicam boa parte de suas variáveis a aspectos relacionados à estrutura e aos sistemas organizacionais. A análise dos modelos traz uma contribuição relevante quanto à necessidade do alinhamento tanto dos elementos formais (como estrutura, processos, indicadores, sistemas de recompensa), quanto dos informais (como pessoas, cultura, sistema de liderança). A eficácia na implementação da estratégia vai depender da forma como a organização é gerenciada, da flexibilidade da estrutura e dos sistemas e do grau de envolvimento das pessoas com as mudanças propostas. Desta forma, os modelos buscam mecanismos de desdobramento e comunicação da estratégia, e constroem métricas e/ou ferramentas de diagnóstico que permitam monitorar o grau de alinhamento e a evolução da estratégia em curso.

Quadro 2: Quadro analítico distintivo dos modelos de alinhamento estratégico interno.

\begin{tabular}{|c|c|c|c|c|}
\hline Componentes & $\begin{array}{c}\text { Modelo } 1 \\
\text { Labovitz e Rosansky } \\
\text { (1997) }\end{array}$ & $\begin{array}{c}\text { Modelo } 2 \\
\text { Balanced Scorecard } \\
\text { Kaplan e Norton } \\
(1992,1993,1996 \text { e 2000) }\end{array}$ & $\begin{array}{c}\text { Modelo } 3 \\
\text { Hambrick e Cannella } \\
\text { (1989) }\end{array}$ & $\begin{array}{c}\text { Modelo } 4 \\
\text { Beer e Eisenstadt } \\
\text { (1996 e 2000) }\end{array}$ \\
\hline Visão do alinhamento & $\begin{array}{c}\text { processo de integração } \\
\text { dos recursos e sistemas } \\
\text { da organização em torno } \\
\text { do propósito essencial } \\
\text { dos negócios }\end{array}$ & $\begin{array}{c}\text { processo de } \\
\text { desdobramento da } \\
\text { estratégia em um } \\
\text { conjunto equilibrado de } \\
\text { medidas de desempenho }\end{array}$ & $\begin{array}{l}\text {. processo gerencial, } \\
\text { previamente planejado } \\
\text { e conduzido pelo } \\
\text { estrategista }\end{array}$ & $\begin{array}{l}\text { processo de mudança } \\
\text { sistêmico e participativo }\end{array}$ \\
\hline $\begin{array}{c}\text { Modelo para } \\
\text { implementação }\end{array}$ & $\begin{array}{l}\text { estabelecimento de } \\
\text { indicadores críticos } \\
\text { de sucesso, metas e } \\
\text { atividades definidos } \\
\text { a partir do propósito } \\
\text { essencial }\end{array}$ & $\begin{array}{l}\text { construção de mapas } \\
\text { estratégicos (balanced } \\
\text { scorecards) }\end{array}$ & $\begin{array}{l}\text { etapas (trilha) a serem } \\
\text { conduzidas pelo } \\
\text { estrategista }\end{array}$ & $\begin{array}{l}\text { diagnóstico de ajuste } \\
\text { organizacional e plano } \\
\text { de implementação }\end{array}$ \\
\hline Elementos do modelo & $\begin{array}{c}\text { alinhamento entre } \\
\text { quatro elementos em } \\
\text { duas dimensões } \\
\text {. estratégia e } \\
\text {. pessoas (alinhamento } \\
\text { vertical) } \\
\text {. clientes e } \\
\text {. processos } \\
\text { (alinhamento horizontal) }\end{array}$ & $\begin{array}{c}\text { indicadores balanceados } \\
\text { em quatro perspectivas: } \\
\text {. financeira } \\
\text {. clientes } \\
\text {. processos } \\
\text {. aprendizado e } \\
\text { crescimento }\end{array}$ & $\begin{array}{l}\text { amplitude de entradas } \\
\text { na formulação } \\
\text {. obstáculos à } \\
\text { implementação } \\
\text {. influências relevantes } \\
\text { na implementação } \\
\text {. convencimento a } \\
\text { respeito da estratégia }\end{array}$ & $\begin{array}{c}\text {. capacidades } \\
\text { organizacionais } \\
\text {. contexto (ambiente } \\
\text { organizacional e } \\
\text { competitivo) } \\
\text { estrutura e políticas de } \\
\text { Recursos Humanos } \\
\text {. opções estratégicas }\end{array}$ \\
\hline $\begin{array}{l}\text { Processos gerenciais } \\
\text { para implementação }\end{array}$ & $\begin{array}{c}\text { PDR } \\
\text {. planejamento } \\
\text { desenvolvimento } \\
\text {. revisão }\end{array}$ & $\begin{array}{c}\text {. tradução da visão } \\
\text {. comunicação e ligação } \\
\text {. planejamento do } \\
\text { negócio } \\
\text { feedback e aprendizado }\end{array}$ & $\begin{array}{c}\text { definição da direção } \\
\text { estratégica } \\
\text {. definição de planos } \\
\text {. negociação } \\
\text {. convencimento } \\
\text {. monitoramento e } \\
\text { controle }\end{array}$ & $\begin{array}{c}\text {. ter uma declaração } \\
\text { sobre as diretrizes } \\
\text { estratégicas } \\
\text {. conhecer barreiras e } \\
\text { forças à implementação } \\
\text {. desenvolver um } \\
\text { plano integrado para } \\
\text { mudança } \\
\text {. refinar o plano } \\
\text {. implementar }\end{array}$ \\
\hline
\end{tabular}


São também estabelecidos mecanismos de retroalimentação para promover ajustes necessários ao longo do processo de transformação das estratégias em ação. Nesse contexto, os modelos de alinhamento estudados se aproximam de outros modelos com foco em gestão, como a gestão por objetivos (management by objectives - MBO) e gerenciamento pelas diretrizes (hoshin kanri ou policy deployment) (COLE, 1998; LEE; DALE, 2000, WITCHER, 2003; WOOD JR; URDAN, 1994).

Por fim, os modelos se dedicam a explorar o papel da liderança. A própria habilidade de implementar a estratégia é considerada uma fonte de vantagem competitiva. Os modelos atribuem à liderança aspectos como o conhecimento dos obstáculos à implementação (tangíveis e intangíveis) e função proativa na negociação da estratégia, interna e externamente. Adicionalmente, deve garantir o desenvolvimento das capacidades organizacionais, atuando na coordenação, e buscando o comprometimento das pessoas para a execução das estratégias. Finalmente, a liderança deve ser atenta às mudanças ocorridas tanto nas variáveis endógenas quanto nas variáveis exógenas e promover os ajustes necessários com rapidez. Assim como nos modelos selecionados, a literatura destaca esse imperativo. Powell (1992) argumenta que o alinhamento requer que o executivo demonstre uma alta capacidade de integração e que a habilidade de alinhamento organizacional poderia ser considerada um recurso estratégico rentável, capaz de melhorar a performance organizacional. Por outro lado, Eisenhardt (1999) destaca o caráter intuitivo tão necessário para enfrentar com flexibilidade e agilidade as barreiras tangíveis e intangíveis que surgem ao longo do processo estratégico.

\section{CONSIDERAÇÕES FINAIS}

Este trabalho foi idealizado após a identificação da existência de lacunas na literatura quanto à dificuldade de operacionalização do conceito de alinhamento estratégico interno (CYPHER, 1996; NAMAN; SLEVIN, 1993; PRIETO; CARVALHO, 2004a, 2004b; SEMLER, 1997; VENKATRAMAN; CAMILLUS, 1984). Após a busca e revisão nas bases de dados foram identificados quatro modelos de alinhamento.

Os modelos selecionados estão de acordo com a definição de alinhamento proposta neste trabalho (ver Quadro 1) e trazem uma contribuição quanto à definição de elementos a serem ajustados e de processos gerenciais a serem conduzidos no processo de alinhamento interno. Além disso, os modelos apresentam ferramentas para medir o grau de alinhamento (modelo 1), fazer diagnósticos (modelos 1 e 4), bem como sistemas de indicadores de avaliação da evolução das estratégias em curso (modelos 1 e 2).

Uma das contribuições do trabalho é apresentar diferentes visões sobre o alinhamento estratégico interno, representadas pelos modelos estudados. No entanto, as diferentes visões não são excludentes e podem ser combinadas ou priorizadas para ampliar a eficácia na implementação da

\section{Quadro 3: Ações para promover o alinhamento estratégico.}

\section{Estratégia}

- Clareza na definição estratégica

- Definição dos fatores críticos de sucesso

- Estratégia alinhada com as necessidades dos stakeholders

\section{Estrutura/Sistema}

- Desdobrar a estratégia em um conjunto de medidas de desempenho balanceadas

- Comunicar a estratégia por meio do mapa estratégico a todos os níveis da organização

- Ajustar os processos para atender os requisitos dos clientes

- Medir o grau de alinhamento e promover ajustes

- Manter atividades de controle da estratégia

- Alocação de recursos estratégicos

- Sistema de recompensas e reconhecimento

- Ajuste de políticas e programas das subunidades

- Definição da estrutura

- Organização do Trabalho

- Pessoas

- Cultura Organizacional

\section{Ambiente}

- Ter informações abrangentes

- Analisar obstáculos

- Negociar a nova estratégia

\section{Liderança}

- Conhecer antecipadamente os obstáculos à implementação (tangíveis e intangíveis)

- Buscar informação e apoio já no estágio de formulação da estratégia

- Negociar a estratégia interna e externamente

- Desenvolver as capacidades organizacionais de coordenação, comprometimento e competência técnica e interpessoal voltadas à implementação de estratégias

- Ajustar os fatores que influenciam a implementação 
estratégia. Os modelos 1 e 2 representam uma visão mais pragmática do alinhamento, com proposta concreta de envolvimento de toda a organização com a estratégia por meio de um sistema de métricas e mecanismos de diagnóstico e ajustes. A visão deste modelo pode ser ampliada com o entendimento de que um processo de mudança está em ação, de que fatores intangíveis podem representar obstáculos e, também, de que os executivos têm o papel de negociar a estratégia em todos os níveis, aspectos esses mais desenvolvidos nos modelos 3 e 4 .

Um resultado importante oriundo tanto da análise comparativa dos modelos (seção 5.1) quanto da análise dos imperativos do alinhamento (seção 5.2) é a identificação de lacunas entre os modelos, que deixam explícitas as limitações individuais de cada um deles, ao mesmo tempo em que amplia a compreensão sobre a visão do alinhamento e das ações ou etapas que devem fazer parte do alinhamento para a sua concretização.

As lacunas identificadas nesse trabalho podem ser incorporadas aos modelos teóricos e práticos de alinhamento estratégico, que contribuem para ampliar a visão a respeito da eficácia na execução da estratégia, bem como podem aumentar a permeabilidade dos modelos.

As diferentes visões e ações podem ser combinadas ou priorizadas para ampliar a eficácia da implementação da estratégia. A escolha do modelo pode se dar, inclusive, de acordo com o grau de maturidade da organização para conduzir um processo de alinhamento. Além disso, os modelos devem refletir o ambiente em que a empresa está inserida. Em ambientes turbulentos, os modelos de alinhamento devem ser mais flexíveis e menos estruturados, dando maior autonomia ao tomador de decisão em busca de agilidade, enquanto em ambientes mais estáveis é possível valer-se de modelos bem estruturados para suporte à decisão, dado que as variáveis e suas relações podem ser modeladas (CARVALHO; LAURINDO, 2007). Por exemplo, o modelo 4 de diagnóstico e implementação da estratégia poderia ser considerado para promover o alinhamento em empresas onde fatores de natureza intangível, tais como capacidades organizacionais de coordenação e formulação da estratégia, filosofias e valores da administração, além das próprias alternativas estratégicas em relação ao contexto organizacional, precisassem ser prioritariamente questionados e revisados. Já a adoção do modelo 3 parece ser compatível com empresas que tenham um certo conhecimento a respeito do processo de formulação e implementação da estratégia, nas quais o estrategista tende a ter uma visão ampla das variáveis que influenciam a formulação da estratégia e cuide da sua implementação por meio do exercício de atividades, como a alocação de recursos, a remoção de obstáculos e o convencimento a respeito da estratégia.

\section{modelo teria como vantagem reduzir a uma dimensão tratável e administrável o problema} da implementação da estratégia.

Este estudo não se esgota com a identificação das ações e etapas aqui identificadas, que podem ser aprofundadas por meio de uma revisão de literatura pontual sobre cada elemento identificado (exemplo: PRIETO, 2006). As restrições do estudo também dizem respeito à delimitação ao construto de alinhamento interno, quando na prática não é possível dissociar a formulação da implementação da estratégia. Uma sugestão para trabalhos futuros é ampliar a base teórica, identificando outros modelos de alinhamento e outras contribuições para compreensão de como desenvolver o alinhamento estratégico, incorporando a dimensão da formulação da estratégia e ajuste ambiental, ou seja, pesquisar conjuntamente o alinhamento externo e interno. Outra sugestão é confrontar as ações identificadas como sendo relevantes para promover o alinhamento com a prática adotada por empresas.

Conforme destacam Hrebiniak e Joyce (2001), trata-se de um tema complexo e os modelos permitem explorar as relações lógicas entre as variáveis e prever os resultados. Por outro lado, os modelos, ao reduzir a realidade à dimensão tratável, promovem simplificações e por isso devem ser vistos dentro de contextos bem delineados, uma vez que a generalização pode conduzir a inadequações.

\section{Artigo recebido em 27/10/2007 Aprovado para publicação em 26/03/2009}




\section{REFERÊNCIAS}

BARDIN, L. Análise de conteúdo. Lisboa: Edições 70, 2000, 225 p.

BARNEY, J. Firm resources and sustained competitive advantage. Journal of Management, v. 17 , n. 1, p. 99-120, 1991

BEER, M.; EISENSTAT, R. A. Developing an organization capable of implementing strategy and learning. Human Relations, v. 49, n. 5, p. 597-603, 1996.

. The silent killers of strategy implementation and learning. Sloan Management Review, v. 41, n. 4, p. 29-40, 12 p., Summer, 2000.

BOWER, J. L. Solving the problems of business planning. Journal of business strategy, v. 2, n. 3 , p. 32-44, 1982.

CAMPBELL, A.; ALEXANDER, M. What's wrong with strategy? Harvard Business Review, p. 4251, Nov.-Dec., 1997.

CARVALHO, M. M.; LAURINDO, F. J. B. Estratégia Competitiva: dos conceitos à implementação. Editora Atlas, 2. ed, 2007.

CHANDLER, A. D. Strategy and structure: chapters in the history of the industrial enterprise. Cambridge, MIT Press, 1962.

CHAKRAVARTHY, B. S.; DOZ, Y. Strategy process research: focusing on corporate selfrenewal. Strategic Management Journal, v. 13, n. 8, p. 5-14, 1992.

CHRISTENSEN, C. M. Making strategy: learning by doing. Harvard Business Review, p. 141-156, Nov./Dec., 1997.

COLLIS, D. J.; MONTGOMERY, C. A. Competing on resources: strategy in the 1990s. Harvard Business Review, p. 118-128, Jul./Ago.,1995.

COURTNEY, H.; KIRKLAND, J.; VIGUERIE, P. Strategy under uncertainty. Harvard Business Review, p. 67-79, Nov./Dec., 1997.

CYPHER, S. R. The alignment project - researching organizational alignment: how leadership and management teams manage the deployment and alignment of improvement efforts throughout their organizations. Blacksburg:
The Performance Center, Virginia Polytechnic Institute and State University, July, 1996.

DAY, G. S. Creating a market-driven organization. Sloan Management Review, v. 41, n. 1, p. 11-22, 1999.

DESS, G. G. Consensus on strategy formulation and organizational performance: competitors in a fragmented industry. Strategic Management Journal, v. 8, n. 3, p. 259-277, 1987.

EISENHARDT, K. M. Strategy as strategic decision making. Sloan Management Review, p. $65-72,1999$.

EISENHARDT, K. M.; SULL, Donald M. Strategy as simple rules. Harvard Business Review, p. 107-112, Jan., 2001.

FISCHMANN, A. A. Implementação de estratégia: identificação e análise de problemas. São Paulo: Universidade de São Paulo, USP, Brasil, 1987.

FLOYD, S. W.; WOOLDRIDGE, B. Managing strategic consensus: the foundation of effective implementation. Academy of Management Executive, v. 6, n. 4, p. 27-39, 1992.

FREEDMAN, $M$ The genius is in the implementation. Journal of Business Strategy, p. 26-31, Mar./Apr., 2003.

FUCHS, P. H. et al. Strategic integration: competing in the age of capabilities. California Management Review, v. 42, n. 3, p. 118-145, 2000.

GALBRAITH, J. R.; KAZANJIAN, R. K. Strategy implementation: structure, systems and process. St Paul: West Pub., 1986.

HAMBRICK, D. C; CANNELLA JR., A. A Strategy implementation as substance and selling. Academy of Management Executive, v. III, n. 4, p. 278-285, 1989.

HREBINIAK, L. G.; JOYCE, W. F. Implementing Strategy: An appraisal and agenda for future research. In: MICHAEL HITT, R. EDWARD FREEMAN and JEFFREY HARRISON (eds), Handbook of Strategic Management (Blackwell Business, 2001), p. 602-626.

HOLMBURG, C.; KROHMER, H.; WORKMAN, J. P. JR. Strategic consensus and performance: The role of strategy type and market-related dynamism. Strategic Management Journal, 20 p. 339-357, 1999.

KAPLAN, R. S.; NORTON, D. P. The balanced scorecard: measures that drive performance. Harvard Business Review, v. 70, n. 1, p. 71-79, 1992.

Putting the balanced scorecard to work. Harvard Business Review, v. 71, n. 5, p. 134-142, 1993

Using the balanced scorecard as a strategic management system. Harvard Business Review, v. 74, n. 1, p. 75-85, 1996

Having Trouble With Your Strategy? Then map it. Harvard Business Review, v. 78, issue 5 , p. 167-176, 2000 .

KILMANN, R. H. A completely integrated program for creating and maintaining organizational success. Organizational Dynamics, v. 18 , n. 1, p. 5-19, Summer, 2001.

LABOVITZ, G.; ROSANSKY, V. The power of alignment: how great companies stay centered and accomplish extraordinary things. EUA: John Wiley e Sons, 242 p.,1997.

MALINA, M. A.; SELTO, F. H. Communicating and controlling strategy: an empirical study of the effectiveness of the balanced scorecard. Journal of Management Accounting Research, v.13, p. 47-147, 2001

McCONKEY, D. D. Planning in a changing environment. Business Horizons, v. 31, n. 5 , p. 64-72, Sep.-Oct., 1988.

MILES, R. E.; SNOW, C. C. Fit, Failure and the hall of fame. California Management Review, v. 26, n. 3, p. 10-28, 1984

MILLER, D. The genesis of configuration. Academy of Management Review, v. 12, n. 4 , p. 686-701, 1987.

MINTZBERG, $H$. The fall and rise of strategic planning. Harvard Business Review, p. 107-114, Jan./Feb., 1994.

NADLER, D.; TUSHMAN, M. L. A model for diagnosing organizational behavior. Organizational Dynamics, v. 19, n. 2, p. 35-51, 17p, Autumn, 1980. 
NAMAN, J. N.; SLEVIN, D. Entrepeneurship and the concept of fit: a model and empirical tests. Strategic Management Journal. v. 14, n. 2, p. 137-153, 1993.

NEWPORT, S.; DESS, G. G.; RASHEED, A. M. A. Nurturing strategic coherency. Planning Review. v. 19, n. 6, p. 18-47, 1991.

NOHRIA, N.; JOYCE, W.; ROBERSON, B. What really works. Harvard Business Review, p. 42-52. July, 2003.

PORTER, M. E. How competitive forces shape strategy. Harvard Business Review, p. 137-145, 1979.

POWELL, T. C. Organizational alignment as competitive advantage. Strategic Management Journal, v. 13, n. 2, p. 119-134, 1992.

PRIEM, R. L. Top management team group factors, consensus, and firm performance. Strategic Management Journal, v. 11, n. 6, p. 469-478, 1990

PRIETO, V. C. Análise de modelos de alinhamento estratégico interno. São Paulo: 2006. Dissertação (Mestrado em Engenharia de Produção). Programa de pós graduação em Engenharia de Produção. Escola Politécnica da Universidade de São Paulo. Departamento de Engenharia de Produção.
PRIETO, V. C.; CARVALHO, M. M. Análise do Alinhamento Estratégico e o Impacto nos Resultados no Setor de Medicina Diagnóstica. In: ENCONTRO DA ASSOCIAÇÃO NACIONAL DE PÓS-GRADUAÇÃO E PESQUISA EM ADMINISTRAÇÃO, 28, 2004, Curitiba, 2004. Anais ANPAD, 2004a.

Diagnóstico de alinhamento estratégico: estudo de múltiplos casos. In: ENCONTRO NACIONAL DE ENGENHARIA DE PRODUÇÃO, 24, 2004. Florianópolis, 2004. Anais ENEGEP, 2004b.

SEMLER, S. W. Systematic agreement: a theory of organizational alignment. Human Resource Development Quarterly, v. 8, n. 1, p. 23-40, 1997.

SIGGELKOW, N. Change in the presence of fit: the raise, the fall and renaissance of $\mathrm{Liz}$ Chairbone. Academy of Management Journal, v. 44 , n. 4 , p. $838-857,2001$.

STEPANOVICH P. L.; MUELLER, J. D. Mapping strategic consensus. Journal of Business and Management, v. 8, n. 2, p. 147-164, 2002.

VAN DE VEN, A. H. Suggestions for studying strategy process: a research note. Strategic Management Journal, v. 13, n. 8, p. 169-188, 1992.
VASCONCELOS, F. C.; CYRINO, Á. B. Vantagem competitiva: os modelos teóricos atuais e a convergência entre estratégia e teoria organizacional. Revista de Administração de Empresas, São Paulo, v. 40, n. 4, p. 20-37, 2000.

VENKATRAMAN, N. Performance implications of strategic coalignment: a methodological perspective. Journal of Management Studies, v. 27, n. 1, p. 23-41, 19 p., 1990.

VENKATRAMAN, N.; CAMILLUS, J. C. Exploring the concept of 'fit' in strategic management. Academy of Management Review, v. 9, n. 3, p. 513-525, 1984.

WEBER, R. P. Basic content analysis. 2. ed., 95 p., London: Sage, 1990.

WERNERFELT, B. A resource-based view of the firm. Strategic Management Journal, n. 5, p. 171-180, 1984.

ZAJAC, E. J.; KRAATZ, M. S.; BRESSER, R. $K$. F. Modeling the dynamics of strategic fit: A normative approach to strategic change. Strategic Management Journal, v. 21, n. 4, 2000.

\section{SOBRE OS AUTORES}

\section{Vanderli Correia Prieto}

Escola Politécnica da USP / Mackenzie - Centro de Ciências Sociais Aplicadas

End.: Av. Prof. Almeida Prado, 128 Tr. 2 Biênio, 2o andar - 05508-900 - São Paulo - SP - Brasil

Tel.: (11) 3091-5363 Fax (11) 3091-5399

E-mail: vanderli.prieto@poli.usp.br; vprieto@mackenzie.br

\section{Marly Monteiro de Carvalho}

Escola Politécnica da USP

End.: Av. Prof. Almeida Prado, 128 Tr. 2 Biênio, 2o andar - 05508-900 - São Paulo - SP - Brasil

Tel.: (11) 3091-5363 r. 303 Fax 3091-5399

E-mail:marlymc@usp.br

\section{Adalberto Américo Fischmann}

FEA/USP

End.: Av. Professor Luciano Gualberto, 908 - Sala E-112 - Butantã - 05508-900 - Sao Paulo - SP - Brasil

Tel.: (11) 3091-5835 Fax: (11) 3091-5834

E-mail: aafischm@usp.br 